\title{
Halogen-free, phosphorus decorated, bio-waste derived nanocomposite for highly efficient flame retardant for cotton fabric
}

\author{
Pratik S. Dhumal \\ ICT: Institute of Chemical Technology \\ Kshama D. Lokhande \\ ICT: Institute of Chemical Technology \\ Madhuri A. Bhakare \\ ICT: Institute of Chemical Technology \\ Mahesh P. Bondarde \\ ICT: Institute of Chemical Technology \\ Surajit Some ( $\nabla$ sr.some@ictmumbai.edu.in ) \\ ICT: Institute of Chemical Technology
}

\section{Research Article}

Keywords: Graphene, Flame retardancy, Thermal analysis, Eggshell, and Bio-waste

Posted Date: January 24th, 2022

DOI: https://doi.org/10.21203/rs.3.rs-1236283/v1

License: (9) This work is licensed under a Creative Commons Attribution 4.0 International License. Read Full License 


\section{Abstract}

We have prepared an efficient flame retardant composite using biowaste derived phosphorous groups decorated graphene supported nanomaterial. The eggshell was utilized as a source of calcium carbonate, which was converted to monocalcium phosphate (CP) by phosphoric acid treatment. As-prepared monocalcium phosphate was functionalized with graphene to prepare graphene functionalized monocalcium phosphate (GCP). The GCP-coated fabric didn't ignite during the flame test and sustained more than 600 s on continuous exposure to flame without changing its initial length and shape. Whereas, graphene oxide (GO), and CP coated cotton fabric burnt out very easily within a short time. The synthesized GCP coated cotton fabric also confirmed efficient flame retardant property with a high limiting oxygen index (34.1) and char length of $2.5 \mathrm{~cm}$ was generated from the VFT test. This facile method enables an easy process for mass production of cost-effective, bio-waste derived nanomaterial for a significantly highly efficient candidate for different applications in sustainable chemistry, including flame-retardant application.

\section{Introduction}

Fire is the major revolutionary discovery of humans with a two-edged sword in that it is used in almost every aspect of daily life and industry, but it also causes significant damage. Every year approximately $0.2 \%$ of the global economy is lost due to fire disasters without counting its reconstruction and other medical-related issues (Li et al., 2011; Chen et al., 2015; Li et al., 2010). The primary benefit of flamerelated research is the development of highly effective materials capable of protecting both human life and society. Among the various flame retardants available, the fabric containing flame retardants is gaining popularity due to its mechanical properties and lack of comfort (Weil et al., 2008; Horrocks et al., 2005). These fabrics are used for soft furnishings, and unmodified cotton fabric are highly flammable ( $\mathrm{Li}$ et al., 2011; Devaux et al., 2002; Alongi et al., 2014) As a result, the way of making fabric flame retardant is surface modification. Surface modification is cost-effective, simple, frequently utilized, and possess an enormous practical approach (Wang et al., 2020; Zhang et al., 2021; Kim et al., 2014). The choice of the flame retardant is also playing an important role. Originally, boron, silicon, and halogen-based flame retardants were widely employed, but they were phased out because they emit dangerous chemicals into the environment causing deleterious effects on human health's (Wakelyn et al., 2007; Hsiue et al., 1999;

Mercado et al., 2006). For the surface modification, dipping (Xu et al., 2019) and layer-by-layer (LBL) (Li et al., 2010; Laachachi et al., 2011) self-assembly were utilized as simple and extensive approaches to improve the flame retardancy of cotton fabric. In that, the cotton fabrics were originally treated with intumescent flame retardant coatings by Li et al. (Li et al., 2011) This sort of coating is extremely effective, beneficial, and versatile in the fabrication of flame retardant coatings on a variety of substrates (Li et al., 2010; Laachachi et al., 2011; Laufer et al., 2012).

The eggshell employed as a bio-filler was commonly utilized as an intumescent flame retardant coating because it includes a high quantity of calcium carbonate $\left(\mathrm{CaCO}_{3}\right)$, i.e., about $95 \%$ while $5 \%$ of other organic components. Intumescent fire-retardant coating, which is largely composed of an intumescent 
fire-retardant system, a synergist, a binder, and an auxiliary agent, is considered as one of the most effective materials for shielding substrates from fire hazards (Yan et al., 2018). Researchers are interested in using polymer because of its simple compatibility with bio-filters, fire resistance, and mechanical characteristics (Yew et al., 2018). The intumescent flame retardant has lately become a pandemic FR for polymers because of its low smoke, halogen-free, low toxicity, and excellent efficiency (Xueying et al., 2015; Zhang et al., 2018). Researchers have been drawn to developing novel intumescent flame retardant systems using bio-based flame retardants like starch (Olivares et al., 2019; Oassaure et al., 2019), chitosan (Cheng et al.,), and lignin (Song et al., 2016; Liu et al., 2016). Acid was widely utilized to improve the flame retardant characteristic of bio-filler-containing products (Yeh et al., 1995; Duquesne et al., 2004). These flame retardants mainly consist of acid source, blowing agent, and binder. When these types of flame retardants come in contact with the flame, they produce black smoke, heat, and toxic gases (Chen et al., 2016). Also, such type of flame retardants has a limited scope of application, which means they cannot be applicable on cotton fabric.

In addition to the eggshell, seashell (Moustafa et al., 2017), clamshell (Li et al., 2020), and conch shell (Wanf et al., 2021) were utilized as bio-fillers, along with various acid, polymer, and binder sources, to increase the flame retardant characteristics of the composite. The application of these chemicals enhances flame retardant efficiency while also protecting the composition. The usage of these compounds (chemicals) to emphasize the flame retardant characteristic of the composite has a very hazardous effect on the environment and human. These sorts of composites may create health issues such as allergic reactions, skin inflammation, and so on (Tesghai et al., 2019). There is a need to modify the bio-filler in such a way that it should take care of all the aspects of ecology with the enhanced flame retardant property.

Long-lasting phosphorus-containing flame retardant materials have fastened attention due to their efficacy, such as low toxicity, reducing the volatility of fuels, producing the carbon-based char, lowering the pyrolysis temperature, wash fastness resistivity and reducing the afterglow. Phosphorus-containing flame retardants benefit from being effective in both the gaseous and condensed phases by generating chain reactions and producing a shield through char formation, respectively (Fang et al., 2020; Dhumal et al., 2022., Yun et al., 2011). The phosphorous functionalization was done in various techniques, including sponges, poly cables, and textiles (Chen et al. 2012). The phosphorous functions can dehydrate cellulose and increase char formation (Wicklein et al., 2015; Song et al., 2017). Many researchers used the phosphorous-graphene strategy for efficient flame retardancy such as DES functionalized (Pethsangave etal., 2017), polymer-based (Pethsangave et al., 2019), GO in ABS (Higginbotham et. Al., 2009), and FRGQD (Khose et al., 2018). Graphene is forthcoming as a very attractive support material for flame retardation systems. It is a perfect fit for the skeleton of flame retardant materials due to its large surface area, high oxygen-rich active functionalities, low toxicity potential and thermal stability. As a result, society continues to demand efficient materials that are simple to produce, environmentally benign, cheaper in cost, and gentle in nature. According to the literature, the modified bio fillers-based flame retardant may be enhanced by the functionalization of phosphorous groups in the presence of graphene as a high surface area containing a suitable carbon-based skeleton. 
As a result of the need for an environmentally friendly, easy-to-prepare flame retardant nanocomposite, we synthesized one from biowaste. In this, the biowaste (eggshell) was treated with phosphoric acid before being reacted with graphene oxide to prepare a nanocomposite for flame retardant applications. Eggshell was converted to monocalcium phosphate by phosphoric acid treatment, which was functionalized with graphene to prepare graphene functionalized monocalcium phosphate (GCP). The prepared composite can sustain in the continuous flame for more than $10 \mathrm{~min}$ (600 s) as compared to the blank cotton fabric (5 s), coated fabric GO (15 s), and CP coated fabric (10 s). The coating of the GCP is a very simple and easy method that requires a concise time. Compared to the blank cotton fabric, GO coated cotton fabric, and CP coated fabric, the produced composite can maintain the flame for more than 10 minutes.

\section{Experimental}

\subsection{Material and Methods}

Graphite powder (325 mesh, Alfa Aeasar), sulphuric acid (98\% assay with $99 \%$ purity, Merck), hydrochloric acid, hydrogen peroxide (30\%, S D Fine Chemicals, Ltd.), potassium permanganate ( $99 \%$, Alfa Aeasar), and ethanol (99.5\%, Merck). Chemicals and solvents were used as obtained without further purifications. Eggshell was collected from the local canteen.

\subsection{Preparation of graphene oxide (GO):}

As mentioned in the preceding paper, the graphene oxide was synthesized using a modified Hummers technique (Dhumal et al., 2021).

\subsection{The preparation of monocalcium phosphate (CP)}

The waste eggshell was collected as a source of $\mathrm{CaCO}_{3}$ from the local canteen and washed several times with water. The washed shells were dried at room temperature first, then in an oven at $80^{\circ} \mathrm{C}$ for $6 \mathrm{hrs}$. To make powder, the dried shells were crushed in a grinder. This eggshell powder was then mixed in a 1:2 ratio with the phosphoric acid solution, as shown in equation 1 . Subsequently, the solution was stirred for an hour and water was added to this solution. After that, the solution was filtered, and the white solid was collected. This white mass was then dried in an oven at $100^{\circ} \mathrm{C}$ for an overnight period.

$\mathrm{CaCO}_{3}+2 \mathrm{H}_{3} \mathrm{PO}_{4} \rightarrow \mathrm{Ca}\left(\mathrm{H}_{2} \mathrm{PO}_{4}\right)_{2}+\mathrm{H}_{2} \mathrm{O}+\mathrm{CO}_{2} \ldots(1)$

\subsection{The preparation of the GCP}

GO (50 mg) was dispersed in deionized water $(50 \mathrm{~mL})$ for GCP preparation. $100 \mathrm{mg}$ of CP were dissolved in $1 \mathrm{~N} \mathrm{HCL}$ solution $(15 \mathrm{~mL}$ ). Both solutions were poured into a $250 \mathrm{~mL}$ round bottom flask and stirred at $85^{\circ} \mathrm{C}$ for $2 \mathrm{~h}$. The reaction mixture was cooled at room temperature after $2 \mathrm{~h}$. of stirring and used as GCP for further experiment. 


\section{Results And Discussion}

X-ray photoelectron spectroscopy (XPS), Fourier transform infrared spectroscopy (FT-IR), thermogravimetric analysis (TGA), X-ray diffraction (XRD), and scanning electron microscopy was used to characterize the as-prepared materials (SEM).

The surface morphology of the as-prepared materials was studied by scanning electron microscopy (SEM). In the case of GO, it has a thin, wrinkled sheet-like structure (Some et al., 2012). The morphological changes have been traced by performing SEM of GCP coated material before and after the fire test and SEM pictures are displayed in Figure 1. The shape of the GCP in these images makes it appear like the $\mathrm{CPs}$ are evenly dispersed on the graphene surface. The CP particles are clustered together in a form that resembles a bundle (Figure 1a). The morphology of the $\mathrm{CP}$ alters following the flame test as it transforms into granular form, which is interesting. It might be because when the CP is exposed to heat, it forms a stable crystal structure in the form of granules or spheres, as shown in Figure 1b (Welzel et al., 2004).

Furthermore, as shown in Figure 2a, the cotton fabric was being uniformly coated by the composite throughout the surface, and its elemental mapping contains carbon, phosphorous, oxygen, and calcium (Figure 2c). In addition, the cotton fabric retained its morphology and structure (Figure $2 b$ ) as well as its elemental mapping, depicting an equal distribution of the carbon, phosphorous, oxygen, and calcium elements, as shown in Figure 2d. The EDX mapping of the GCP composite was described on the Table S1.

XPS analysis was used to study the surface electronic state and atomic composition of the composite. The surface electrical state of the composite and its atomic composition was investigated using XPS analysis.

The GCP has a high oxygen content i.e., the $\mathrm{C} / \mathrm{O}$ ratio of 0.95 as shown in Table S1. In the spectra survey of GCP (Figure 3a), the C1s, 01s, P2p, P2s, and Ca2p peaks were seen. At 531.2 and $533.2 \mathrm{eV}$, respectively, the $01 \mathrm{~s}$ spectra (Figure $3 \mathrm{~b}$ ) comprise the $\mathrm{C}=\mathrm{O} / \mathrm{P}=\mathrm{O}$ and $\mathrm{C}-\mathrm{O} / \mathrm{P}-\mathrm{O}$ bonding. The GCP's $\mathrm{C} 1 \mathrm{~s}$ spectra (Figure 3c) reveals a strong peak for C-C bonding at $284.6 \mathrm{eV}$, a peak for C-O and C-P at $285 \mathrm{eV}$, and a peak for C-O and C-P at $285 \mathrm{eV}$. The existence of P2p and P2s at 133.2 and $134.6 \mathrm{eV}$, respectively, is confirmed in the GCP spectra, as shown in Figure 3d (Kim et al., 2014; Blundell et al., 2014).

The TGA was performed in an air environment with a flow rate of $20^{\circ} \mathrm{C}$ to assess the thermal stability of the GCP composite. Because of its thermal stability, there is no notable change in weight in the TGA spectra of $\mathrm{CP}$ and calcium carbonate (Figure 4a). Both curves have excellent thermal stability, with weight loss ranging from $15-20 \%$ in each case. The first loss was noticed in the GCP curve at $100^{\circ} \mathrm{C}$ owing to the loss of intercalated water molecules. After that, at $240^{\circ} \mathrm{C}$, a second weight loss was observed due to the burning of the carbon skeleton (Ylmaz et al., 2004). The GCP has lost about $41 \%$ of its total weight, demonstrating the composite's thermal stability. The crystallite structure of the GCP composite material has shown by the XRD pattern (Figure $4 \mathrm{~b}$ ). The usual graphene peak at $26.2^{\circ}$ and the peak at $32.2^{\circ}$ 
suggest the presence of CP crystals (Kamalanathan et al., 2014). Figure S1 depicts the graphene oxide XRD pattern, which exhibits a characteristic peak at $10^{\circ}$.

The FT-IR spectra of GO revealed the following peaks as shown in Figure 5. The peak indicates the wide vibration of the $\mathrm{O}-\mathrm{H}$ band at $3424 \mathrm{~cm}^{-1}$. The presence of $\mathrm{C}=0$ stretching of acid and $\mathrm{C}=\mathrm{C}$ stretching vibration of aromatic carbon atoms was verified by the appearance of peaks at 1728 and $1623 \mathrm{~cm}^{-1}$, respectively. Peaks were found at 1412 and $1053 \mathrm{~cm}^{-1}$, suggesting $\mathrm{C}-\mathrm{H}$ bending and $\mathrm{C}-\mathrm{O}$ stretching, respectively (Higginbotham et. Al., 2009.

In the spectra of the $C P$, the peaks found at 569,950 , and $1092 \mathrm{~cm}^{-1}$ attributed to the vibrations of phosphate group PO4 ${ }^{-3}$ (Yelteen et al., 2016). Also, the hydroxy group (-OH) is represented by the steep peak at $3467 \mathrm{~cm}^{-1}$ and the peak at $650 \mathrm{~cm}^{-1}$. Peaks for the phosphate group $\left(\mathrm{PO}^{-3}\right)$ at 599, 977, and $1024 \mathrm{~cm}^{-1}$ in the GCP spectra demonstrate the incorporation of monocalcium phosphate into the graphene core. At $1440 \mathrm{~cm}^{-1}$, the peak for $\mathrm{C}-\mathrm{H}$ stretching was also seen. From the peak at $1627 \mathrm{~cm}^{-1}$, the $\mathrm{C}=\mathrm{C}$ stretching vibration bonding was confirmed.

\section{Flame retardant test of the GCP coated cotton fabric}

An ethanol lamp was utilized to conduct the flame retardant test.

A dipping procedure was used to coat the GCP sample on the fabric. The GO and CP coated cotton fabrics were treated in the same way. The GO coated cotton fabric and blank cotton fabric were tested for the flame experiment in the air atmosphere, as shown in Figure (6 a-c) and Video V1. The GO coated fabric burnt out within $15 \mathrm{~s}$, whereas the blank cotton fabric burnt out within $5 \mathrm{~s}$. The CP coated burnt out in $10 \mathrm{~s}$, exhibited the same phenomena in comparison to other control samples (Figure 6d-f and Video V2). In the case of GCP, the coated fabric did not catch fire for more than 10 minutes (600s) on continuous flame and retained its original shape and size, as illustrated in Figure ( $6 \mathrm{~g}$-i and Video V3). The plausible mechanism of the GCP composite for flame retardancy is shown in Figure S2. As a result, GCP coated fabric was the most promising flame retardant material compared to GO and CP coated cotton fabric. The chemistry of flame retardancy is determined by the recurrence of chemical structural elements and their interaction during combustion. Because of the strong bonding interaction, the energy required to break the sp2 hybridized carbon atom is higher. As a result, any compounds containing the sp2 carbon's strong backbone, are more flame resistant (Walters et al., 2002; Lyon et al., 2009).

To evaluation of the flame retardant property of the GCP composite, the vertical flammability test (VFT) was performed. The test was done as per the ASTMD6413-09 standard method for the flame-resistant test of cotton fabric. The flame height was $38 \mathrm{~mm}$ and the sample dimensions were $30 \times 7.6 \mathrm{~cm}^{2}$. The bottom cut edge of the fabric was subjected to a controlled flame for $12 \mathrm{~s}$, followed by flashing over the surface, burning with flame time, burning with afterglow after flame stop, and char length measured as shown in Figure 6. Table S2 shows the results of the vertical flammability test, which were outstanding, as well as detailed flammability data. The blank fabric lit quickly and burned up completely in less than 
$18 \mathrm{~s}$ (Figure 7a), with flashes throughout the surface. The GO covered fabric was entirely burned out in 11 $s$ (Figure 7b), with flashing across the surface afterwards. The GCP coated fabric did not display flash across the surface and did not burn, although it did have a $2 \mathrm{~s}$ afterglow, resulting in a total burning time of $2 \mathrm{~s}$ (Teli et al., 2017) and measured char formation is $2.5 \mathrm{~cm}$ (Figure 7c). Table S3 summarizes the data and LOI explanations for the composite. Also, table S4 contains a comparative study of the char length of similar materials.

The mechanical property of the flame retardant composite plays important role. Tensile strength of the GCP composite was discussed in the S3. Whereas, the washing fastness of calcium carbonate, $\mathrm{CP}$, and GCP coated cloth/binder before and after washing was analyzed and weight percent loss calculated and mentioned in Figure S5.

\section{Conclusions}

We have synthesized halogen-free, phosphorus functional groups decorated, sustainable, bio-waste derived nanocomposite for efficient flame retardant for cotton fabric. Leftover eggshells were treated with phosphoric acid to prepare monocalcium phosphate, which was functionalized with graphene to prepare GCP. The as-prepared GCP composite coated cotton fabric can prevent a continuous flame for more than $600 \mathrm{~s}$, whereas the CP and GO coated cotton fabrics burn out in 10 and $15 \mathrm{~s}$, respectively, with smoke formation. The major advantage of the GCP coated fabric is to protect its initial shape and size after being exposed to a continuous flame for 10 minutes. The both LOI and vertical flammability test confirmed the GCP's flame retardant properties. The GCP coated fabric has a high LOI value of 34.1, indicating good flame retardant properties. The phosphorus decorated bio filler functionalized graphene nanocomposite was synthesized by using a simple and green approach, which has high efficiency as flame retardancy for cotton fabric. The present study indicates the environmentally benign, sustainable efficient flame retardant nanocomposite deserves exploration for different fabrics.

\section{Declarations}

\section{AUTHOR INFORMATION}

Corresponding Author

* Dr. Surajit Some

E-Mail: sr.some@ictmumbai.edu.

Present Addresses

Department of Speciality Chemical Technology,

Institute of Chemical Technology Matunga, 
Mumbai-400 019, India.

Author Contributions

P.S.D. and S.S. wrote the manuscript, P.S.D. performed experiment, P.S.D., K.D.L., M.A.B. and M.P.B. prepared graphene oxide and nanocomposite. P.S.D. analyzed all data, S.S supervised all work.

\section{Compliance with ethical standards}

Conflict of interest: The authors declare that they have no conflict of interest.

Ethical approval: The authors have no statement about ethical approval. Because, this research does not involve human and animal.

Informed consent: This research does not involve human participants that's why author have no statement about informed consent.

\section{Acknowledgements}

This work was supported by the Chhatrapati Shahu Maharaj Research Training and Human Development Institute (SARTHI), and the CSIR fund (22/0748/17/EMR-II). Pratik Dhumal is thankful to SARTHI for his fellowship.

\section{References}

1. Alongi J, Carosio F, Malucelli G (2014) Current Emerging Techniques To Impart Flame Retardancy to Fabrics: An Overview. Polym Degrad Stab 106:138-149

2. Blundell RK, Licence P (2014) Quaternary ammonium and phosphonium based ionic liquids: a comparison of common anionsm. Phys Chem Chem Phys 16:15278-15288

3. Chen MJ, Shao ZB, Wang XL, Chen L, Wang YZ (2012) Halogen-Free Flame-Retardant Flexible Polyurethane Foam with a Novel Nitrogen-Phosphorus. Flame Retardant Ind Eng Chem Res 51:9769-9776

4. Chen P, Zhang F, Li S, Cheng Y (2016) Smoke suppression properties of epoxy crosslinked structure and intumescent fire retardant in epoxy-based intumescent fire-retardant coating J. Appl Polym Sci 133:8782

5. Chen S, Li X, Li Y, Sun J (2015) Intumescent flame-retardant and self-healing superhydrophobic coatings on cotton fabric. ACS Nano 9:4070-4076

6. Cheng XW, Guan JP, Yang XH, Tang RC, Yao F (2019) A bio-resourced phytic acid/chitosan polyelectrolyte complex for the flame retardant treatment of wool fabric. J Cleaner Prod 223:342349

7. Devaux E, Rochery M, Bourbigot S (2008) Polyurethane/Clay and Polyurethane/POSS Nanocomposites as Flame Retarded Coating for Polyester and Cotton Fabrics. Fire Mater 26:149- 
8. Dhumal PS, Khose RV, Wadekar PH, Lokhande KD, Some S (2021) Graphene-bentonite supported free-standing, flexible membrane with switchable wettability for selective oil-water separation. Sep Purif Technol 266:118569

9. Dhumal PS, Lokhande KD, Bondarde MP, Bhakare MA, Some S (2022) Heat resistive, binder-free 3ddough composite as a. https://doi.org/10.1002/app.52146. highly potent flame-retardant

10. Duquesne S, Magnet S, Jama C, Delobel R (2004) Intumescent paints: fire protective coatings for metallic substrates Surf. Coat Technol 302:180-181

11. Fang F, Huo S, Shen H, Ran S, Wang H, Song P, Fang Z (2020) A bio-based ionic complex with different oxidation states of phosphorus for reducing flammability and smoke release of epoxy resins. Composites Communications 17:104-108

12. Higginbotham AL, Lomeda JR, Morgan AB, Tour JM (2009) Graphite oxide flame retardant polymer nanocomposites. ACS Appl Mater Interfaces 1:2256-2261

13. Horrocks AR, Kandola BK, Davies PJ, Zhang S, Padbury SA (2005) Developments in flame retardant textiles-a review. Polym Degrad Stab 88(1):3-12

14. Hsiue GH, Wang WJ, Chang FC (1999) Synthesis, Characterization, Thermal and Flame-Retardant Properties of Silicon-Based Epoxy Resins. J Appl Polym Sci 73:1231-1238

15. Kamalanathan P, Ramesh S, Bang LT, Niakan A, Tan CY, Purbolaksono J (2014) Synthesis and sintering of hydroxyapatite derived from eggshells as a calcium precursor. Ceram Int 40:1634916359

16. Khose RV, Pethsangave DA, Wadekar PH, Ray AK, Some S (2018) ; Novel approach towards the synthesis of carbon-based transparent highly effective flame retardant Carbon 139:205-209

17. Kim MJ, Jean IY, Seo JM, Dai L, Baek JB (2014) Graphene Phosphonic Acid as an Efficient Flame Retardant. ACS Nano 8:2820-2825

18. Laachachi A, Ball AK, Toniazzo V, Ruch D (2011) Diffusion of Polyphosphates into (Poly(allylamine) montmorillonite) Multilayer Films: Flame RetardantIntumescent Films with Improved Oxygen Barrier. Langmuir 27:13879-13887

19. Laufer G, Kirkland C, Morgan AB, Grunlan JC (2012) Made with Renewable Polyelectrolytes, for Flame-Retardant Cotton. Biomacromolecules 13:2843-2848

20. Li Y, Feng Y, Xu Z, Yan L, Xie X, Wang Z (2020) Synergistic effect of clam shell bio-filler on the fireresistance and char formation of intumescent fire-retardant coatings. Journal of Materials Research and Technology 9(6):14718-14728

21. Li YC, Schulz J, Mannen S, Delhom C, Condon B, Chang S, Zammarano M, Grunlan JC (2010) Flame retardant behavior of polyelectrolyte-clay thin film assemblies on cotton fabric. ACS Nano 4:33253333

22. Li YC, Mannen S, Morgan AB, Chang S, Yang YH, Condon B, Grunlan JC (2011) Intumescent allpolymer multilayer nanocoating capable of extinguishing flame on fabric. Adv Mater 23:3926-3931 
23. Liu L, Huang G, Song P, Yu Y, Fu S (2016) Converting industrial alkali lignin to biobased functional additives for improving fire behaviour and smoke suppression of polybutylene succinate. ACS Sustainable Chem Eng 4:4732-4742

24. Lyon RE, Takemori MT, Safronava N, Stoliarov SI, Walters RN (2009) ; A molecular basis for polymer flammability Polymer 50:2608-2617

25. Song M, Yu GHuang, Fu Y S (2016) Fabrication of green lignin-based flame retardants for enhancing the thermal and fire retardancy properties of polypropylene/wood composites. ACS Sustainable Chem Eng 4:2422-2431

26. Mercado L, Galia M, Reina J (2006) Silicon-containing flame retardant epoxy resins: Synthesis, characterization and properties. Polym Degrad Stab 91:2588-2594

27. Moustafa H, Youssef AM, Duquesne S, Darwish NA (2017) Characterization of bio-filler derived from seashell wastes and its effect on the mechanical, thermal, and flame retardant properties of ABS composites. Polym Compos 38(12):2788-2797

28. Olivares GS, Rabe S, Chavez RP, Calderas F, Schartel B (2019) Industrial-waste agave fibres in flameretarded thermoplastic starch biocomposites. Composites Part B 177:107

29. Passauer $L$ (2019) Thermal characterization of ammonium starch phosphate carbamates for potential applications as bio-based flameretardants. Carbohydr Polym 211:69-74

30. Pethsangave DA, Khose RV, Wadekar PH, Some S (2017) Deep Eutectic Solvent Functionalized Graphene Composite as an Extremely High Potency Flame Retardant. ACS Appl Mater Interfaces 9:35319-35324

31. Pethsangave DA, Khose RV, Wadekar PH, Some S (2019) Novel Approach toward the Synthesis of a PhosphorusFunctionalized Polymer-Based Graphene Composite as an Efficient Flame Retardant. ACS Sustainable Chem Eng 7:11745-11753

32. Some S, Ho SM, Dua P, Hwang E, Shin YH, Yoo H, Kang J, Lee DK, Lee H (2012) Dual functions of highly potent graphene derivative-poly-l-lysine composites to inhibit bacteria and support human cells. ACS Nano 6:7151-7161

33. Song K, Ganguly I, Eastin I, Dichiara AB (2017) Lignin-Modified CarbonNanotube/Graphene Hybrid Coating as Efficient Flame Retardant. Int J Mol Sci 18:2368

34. Teli MD, Pintu P (2017) Development of thermally stable and hygienic colored cotton fabric made by treatment with natural coconut shell extract. J Ind Text 0(00):1-32

35. Tseghai GB, Berhe BT, Wakjira YT (2019) ; Producing fire retardant cotton fabric using chicken eggshell.Journal of textile science \& engineering9(2)

36. Wakelyn PJ, Bertoniere NR, French AD (2007) Thibodeaux D. Cotton fiber chemistry and technology. CRC Press (Taylor and Francis Group), Boca Raton, FL, USA, pp 77-80

37. Walters RN, Lyon RE (2002) Molar group contributions to polymer flammability. J Appl Polym Sci $87: 548-563$ 
38. Wang B, Xu YJ, Li P, Zhang FQ, Liu Y, Zhu P (2020) Flameretardant polyester/cotton blend with phosphorus/nitrogen/siliconcontaining nano-coating by layer-by-layer assembly. Appl Surf Sci 509:145323

39. Wang F, Liu H, Yan L, Feng Y (2021) Comparative Study of Fire Resistance and Anti-Ageing Properties of Intumescent Fire-Retardant Coatings Reinforced with Conch Shell Bio-Filler. Polymers 3(16):2620

40. Weil ED, Levchik SV (2008) Flame retardants in commercial use or development for textiles. J Fire Sci 26:243-281

41. Welzel T, Zaika MW, Epple M (2004) ; Continuous preparation of functionalised calcium phosphate nanoparticles with adjustable crystallinity Chem comm (10):1204-1205

42. Wicklein B, Kocjan A, Alvarez GS, Carosio F, Camino G, Antonietti M, Bergstrom L (2015) Thermally insulating and fire-retardant lightweight anisotropic foams based on Nanocellulose and graphene oxide. Nat Nanotechnol 10:277-283

43. Xu F, Zhong L, Zhang C, Wang P, Zhang F, Zhang G (2019) Novel High-Efficiency Casein-Based P-NContaining Flame Retardants with Multiple Reactive Groups for Cotton Fabrics. ACS Sustainable Chem Eng 7:13999-14008

44. Xueying S, Yuan H, Haiqun C, Xiongjun Y (2015) Study on thermal property, flame retardancy and mechanism of poly (lactic acid)/intumescent flame retardant/NiAl layer double hydroxide nanocomposites. Sci Adv Mater 7:1848-1857

45. Yan L, Xu Z, Wang X (2018); Synergistic effects of organically modified montmorillonite on the flame-retardant and smoke suppression properties of transparent intumescent fire-retardant coatings. Prog Org Coat 2018; 122:107-118

46. Yeh JT, Yang HM, Huang SS (1995) Combustion of polyethylene filled with metallic hydroxides and crosslinkable polyethylene. Polym Degrad Stab 50(2):229-234

47. Yelten A, Yilmaz S (2016); Various parameters affecting the synthesis of the hydroxyapatite powders by the wet chemical precipitation technique. Mater Today Proc 2016; 3:2869-76

48. Yew MC, Kun YM, Huat SL, Ng TC, Durairaj R, Beh JH (2018) Influences of nano bio-filler on the fireresistive and mechanical properties of water-based intumescent coatings. J Prog Org Coating 124:33-40

49. Ylmaz P, öztürk Er E, Bakırdere S (2019) Application of supercritical gel drying method on fabrication of mechanically improved and biologically safe three-component scaffold composed of graphene oxide/chitosan/hydroxyapatite and characterization studies. J Mater Res Technol 8(6):5201-5216

50. Yun L, Cheng LD, Jing Z, Jun SW, Li C, Yu ZW (2011) An efficiently halogen-free flame-retardant longglass-fiber-reinforced polypropylene system. Polym Degrad Stab 96:363-370

51. Zhang AN, Zhao HB, Cheng JB, Li ME, Li SL, Cao M, Wang YZ (2021) Construction of durable ecofriendly biomass-based flame-retardant coating for cotton fabrics. Chem Eng J 410:128361

52. Zhang N, Lu X (2018) thermal and combustion properties of intumescent flame retardant biodegradable poly (lactic acid) composites. Plast Rubber Compos 47:458-467 


\section{Figures}

a)

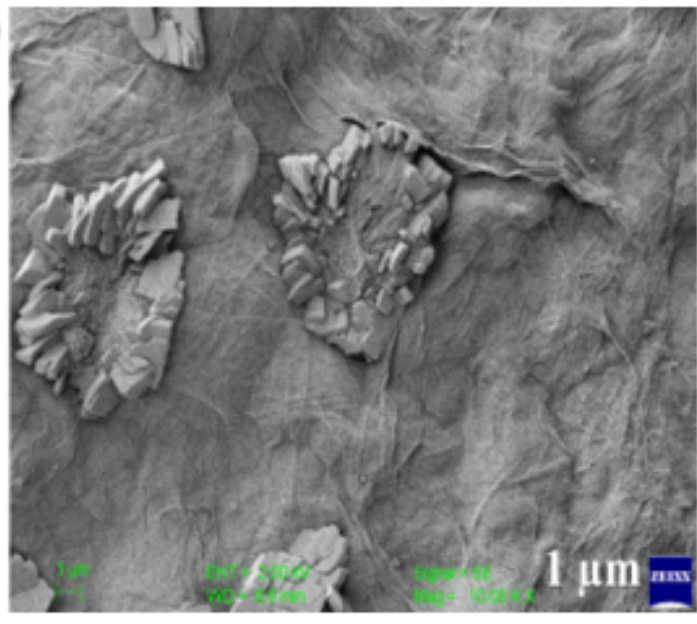

b)

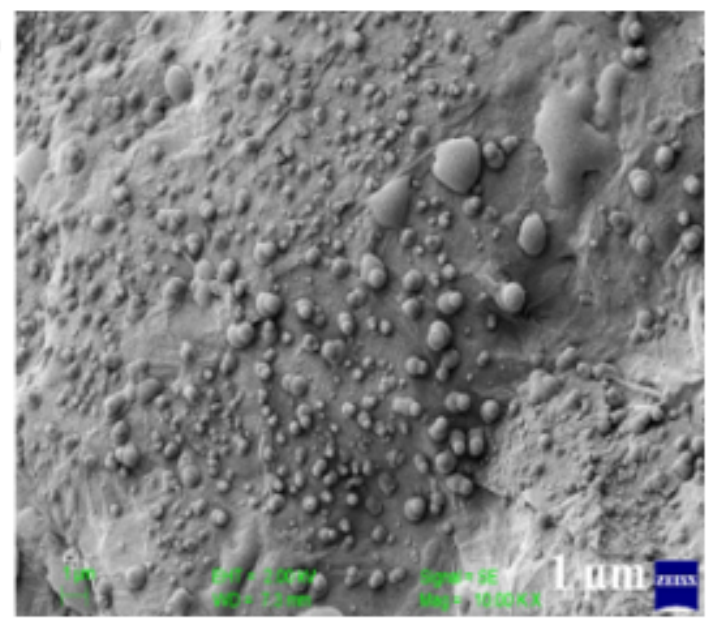

Figure 1

SEM image of the GCP a) before the flame test and $b$ ) after the flame test

Figure 2

SEM images of GCP composite a) before flame and b) after the flame retardant experiment. Corresponding EDX element mapping c) before flame test contains carbon (blue), phosphorous (green), oxygen (orange), and calcium (yellow) and d) after flame test contains carbon (blue), phosphorous (green), oxygen (orange), and calcium (yellow).

\section{Figure 3}

a) XPS survey spectra of the GCP, b) XPS spectra of GCP 01s spectra, c) XPS spectra of GCP C1s spectra, and d) XPS spectra of GCP P2p

\section{Figure 4}

a) TGA graph of calcium carbonate, monocalcium phosphate (CP), and GCP, b) XRD pattern of GCP

\section{Figure 5}


IR spectrum of the GO, CP, and GCP

Figure 6

Snapshots of the flame retardant test of the GO coated cotton fabric (right) with and blank cotton fabric (left) concerning time from (a-c) 0-15s. Snapshots of the flame retardant test of the CP coated cotton fabric (right) with and blank cotton fabric (left) concerning time from (d-f) 0-10s. Snapshots of the flame retardant test of the GCP coated cotton fabric (right) with and blank cotton fabric (left) concerning time from (g-i) 0-600s.

\section{Figure 7}

Snapshot of the vertical flammability test (a) blank cotton fabric, (b) GO coated cotton fabric, and c) GCP coated cotton fabric.

\section{Supplementary Files}

This is a list of supplementary files associated with this preprint. Click to download.

- SupportingInformation.docx

- V1GOcoated.mov

- V2CPcoated.mov

- V3GCPcoated.mp4 